\title{
Imagined own-body transformations during passive self-motion
}

\author{
Michiel van Elk • Olaf Blanke
}

Received: 31 October 2012/ Accepted: 1 February 2013

(C) Springer-Verlag Berlin Heidelberg 2013

\begin{abstract}
Spatial perspective taking is a crucial social skill that underlies many of our everyday interactions. Previous studies have suggested that spatial perspective taking is an embodied process that involves the integration of both motor and proprioceptive information. Given the importance of vestibular signals for own-body perception, mental own-body imagery, and bodily self-consciousness, in the present study we hypothesized that vestibular stimulation due to passive own-body displacements should also modulate spatial perspective taking. Participants performed an own-body transformation task while being passively rotated in a clockwise or counter-clockwise direction on a human motion platform. A congruency effect was observed, reflected in faster reaction times if the implied mental body rotation direction matched the actual rotation direction of the chair. These findings indicate that vestibular stimulation modulates and facilitates mental perspective taking, thereby highlighting the importance of integrating multisensory bodily information for spatial perspective taking.
\end{abstract}

M. van Elk $(\bowtie) \cdot$ O. Blanke

Laboratory of Cognitive Neuroscience, Brain Mind Institute, École Polytechnique Fédérale de Lausanne, Station 19, AI 2101, 1015 Lausanne, Switzerland e-mail: m.vanelk@uva.nl

M. van Elk

University of Amsterdam, Amsterdam, The Netherlands

O. Blanke

Department of Neurology, University Hospital Geneva, Geneva, Switzerland

O. Blanke

Center for Neuroprosthetics, École Polytechnique

Fédérale de Lausanne, Lausanne, Switzerland

\section{Introduction}

An important skill underlying our daily interactions with others is our ability to take the perspective of the other person. For instance, when riding a bike or when driving a car we take into account what other traffic users can see and we adjust our own behavior accordingly. The ability to infer what another person can see marks an important developmental milestone. From about 14 months onwards infants are able to engage in level-1 perspective taking, by representing what another person can and cannot see (Sodian, Thoermer, \& Metz, 2007) and from about 3 years of age children can infer what the world looks like from another person's perspective (Moll \& Meltzoff, 2011). The importance of spatial perspective taking for social cognition is reflected at a language level as well, for instance in expressions like 'putting oneself in the others' shoes' or 'from your point of view'.

Over the last decades, many studies have investigated the functional and neural mechanisms underlying spatial perspective taking. A classical way to study spatial perspective taking is to present participants with a spatial layout representing different objects. Subsequently, participants are placed in a new position or are required to imagine standing in a new position, from where they estimate the location of the different objects. Typically, it is more difficult for participants to correctly estimate the location of the objects after imagined displacements compared to actual displacements (Easton \& Sholl, 1995; Rieser, Garing, \& Young, 1994).

Two hypotheses have been put forward to account for this finding (Avraamides \& Kelly, 2008; May, 2004). Drawing a parallel with classical studies on mental imagery, the mental transformation hypothesis states that the transformation costs in relation to spatial perspective 
taking are primarily related to the switching from the actual location and perspective into the imagined location and spatial perspective (Rieser, 1989). In the absence of proprioceptive input, observers need to engage in a computational process to determine the new position of the objects with respect to their body. The additional computational processing costs associated with imagined compared to actual displacements are reflected in slower reaction times and higher error rates.

In contrast, according to the sensorimotor interference hypothesis, the interference costs during imagined spatial perspective taking are primarily related to sensorimotor conflicts between the imagined and the body-defined perspective (May, 2004). On this account, increased angular disparity between the actual and the imagined spatial perspective results in a stronger discrepancy between the physical and imagined egocentric reference frame, which is reflected in slower response times and higher error rates. This hypothesis accounts for the finding that response latencies and errors increase with increased angular disparity between the imagined and actual spatial perspective (Huttenlocher \& Presson, 1979; Kozhevnikov \& Hegarty, 2001; May, 2004; Zacks \& Michelon, 2005) and that imagined rotations were found to be more difficult than imagined translations (Creem-Regehr, 2003; Rieser, 1989). That is, in the case of translation the conflict between the imagined and the actual egocentric perspective is smaller than in the case of rotation, thereby resulting in less errors.

The sensorimotor interference hypothesis is closely related to the suggestion that spatial perspective taking is an embodied process that involves a mental transformation of the observer's own body (Zacks \& Michelon, 2005). More specifically, it has been proposed that spatial perspective taking involves a process of endogenous motor embodiment, in which a self-initiated emulation of a body movement is used to adopt the imposed perspective (Arzy, Thut, Mohr, Michel, \& Blanke, 2006; Kessler \& Thomson, 2010). This view is supported by the finding that imagined own-body transformation is modulated by one's current body posture (Amorim, Isableu, \& Jarraya, 2006; Kessler \& Thomson, 2010). For instance, Kessler \& Thomson (2010) asked participants to perform a spatial perspective taking task while their body posture could be congruent or incongruent with respect to the imposed perspective. Participants responded faster when their body posture was congruent with the implied rotation direction-as if the body posture gave participants a 'head-start' in the mental transformation process. This finding suggests that spatial perspective taking involves the embodied transformation of one's body to adopt the imposed perspective. This 'embodied transformation account' is further supported by neuroimaging studies showing the involvement of motorrelated areas during perspective taking and indicating that spatial perspective taking involves comparable neural mechanisms that are involved in actual bodily movements as well (Vogeley et al., 2004; Wraga, Shephard, Church, Inati, \& Kosslyn, 2005; Zacks \& Michelon, 2005).

Thus, embodied spatial perspective taking is often construed as an active process, in which the observer puts himself in the shoes of another person by relying on both motor (Huttenlocher \& Presson, 1979; Kozhevnikov \& Hegarty, 2001; Zacks \& Michelon, 2005) and proprioceptive information (Huttenlocher \& Presson, 1979; Kessler \& Rutherford, 2010; Kessler \& Thomson, 2010; Kozhevnikov \& Hegarty, 2001; Tversky \& Hard, 2009; Zacks \& Michelon, 2005). Next to motor and proprioceptive signals, spatial perspective taking may also involve the integration of vestibular information, associated with either active or passive motion of the body. For instance, rotating oneself on a desk chair to take the same visual perspective as a colleague results in a stimulation of the semicircular channels and in the spatial updating of our own body position based on visual and vestibular information (Angelaki \& Cullen, 2008). Rieser, Guth, \& Hill, (1986) have underlined the importance of vestibular information for spatial localization, by showing that blindfolded participants walking a short distance were well able to keep track of their visuo-spatial perspective. Furthermore, recent work has shown the importance of vestibular information for the experienced first person perspective and for bodily selfconsciousness (Ferre, Bottini, \& Haggard, 2011; Ionta et al., 2011; Lopez, Bachofner, Mercier, \& Blanke, 2009; Lopez, Lenggenhager, \& Blanke, 2010). Several studies have shown that due to a visuo-vestibular conflict participants may perceive themselves to be at a different location than the actual position of their body (Ionta et al., 2011; Pfeiffer et al., in prep.; Lopez, Halje, \& Blanke, 2008; Lopez, Heydrich, Seeck, \& Blanke, 2010). Other studies have indicated that when passive own-body displacements are paired with incongruent visual feedback, this can result in a mislocalization of oneself in external space as well (Shinder \& Taube, 2010).

Two recent studies have addressed the relation between vestibular stimulation and imagined own-body transformation more directly (Falconer \& Mast, 2012; Lenggenhager, Lopez, \& Blanke, 2008). It was found that right galvanic vestibular stimulation resulted in slower reaction times during the mental transformation of bodies and objects (Lenggenhager et al., 2008), while caloric vestibular stimulation specifically enhanced the mental transformation of one's own body, but not of body parts or objects (Falconer \& Mast, 2012). Thus, the results of these studies are inconclusive and may be related to the different methodologies used to stimulate the vestibular system. Both caloric and galvanic vestibular stimulation result in an unnatural activation of the vestibular organ (as well as 
other undesired side effects) and the activation of a large network of cortical structures, involving both visual and multisensory association areas (Lopez \& Blanke, 2011). In the present study we propose to use passive whole-body rotations as a more natural way to selectively stimulate the semicircular canals and to investigate the effects of vestibular stimulation on mental own-body transformation.

Thus, given the importance of vestibular information for own-body perception and bodily self-consciousness, in the present study we hypothesized that passive own-body displacements should facilitate spatial perspective taking. To test this hypothesis, we used an own-body transformation task as a well-established paradigm to measure spatial perspective taking (Arzy, Thut, Mohr, Michel, \& Blanke, 2006; Blanke, Ionta, Fornari, Mohr, \& Maeder, 2010; Blanke et al., 2005; Mohr, Blanke, \& Brugger, 2006; Parsons, 1987; Tadi, Overney, \& Blanke, 2009). ${ }^{1}$ In this task participants are required to make laterality judgments regarding the handedness of a marked hand of an avatar presented at different angular disparities. Typically, reaction times and error rates increase with increased angular disparity between the participant's own body position and the avatar's position, reflecting increased effort to mentally put oneself in the avatar's position (Parsons, 1987).

Importantly, in his seminal study on mental body rotation, Parsons, (1987) already showed that participants imagined themselves rotating along the shortest path to match the avatar's body position. Thus, depending on the position of the avatar, participants imagine themselves rotating in a clockwise $(\mathrm{CW})$ or a counter-clockwise direction $(\mathrm{CCW})$. In the present study we exploited this fact by having participants perform a mental body transformation (MBT) task, while undergoing passive wholebody rotations. Participants were passively rotated on a human motion platform in a $\mathrm{CW}$ or a $\mathrm{CCW}$ direction and performed MBTs that implied either a $\mathrm{CW}$ or a $\mathrm{CCW}$ rotation direction. Based on previous studies, showing effects of galvanic and caloric vestibular stimulation on the mental rotation of objects and bodily stimuli (Falconer \& Mast, 2012; Lenggenhager et al., 2008), in the present study we hypothesized that spatial perspective taking would be facilitated if the implied direction of the mental body rotation was the same as the actual passive own-body rotation. Such a finding would extend previous studies showing facilitatory effects of one's own body posture on spatial perspective taking (Amorim et al., 2006; Kessler \& Thomson, 2010) to the domain of vestibular processing.

\footnotetext{
1 As different processes are involved in mental body transformations (Gardner \& Potts, 2011; May \& Wendt, 2012) and subjects often report different strategies (Kessler \& Wang, 2012) we have decided in this manuscript to use the term "mental body transformation" instead of own body transformation.
}

\section{Methods}

\section{Participants}

In total 18 right-handed participants participated in the experiment ( 5 females, mean age $=23.7$ years), all students at the École Polytechnique Fédérale de Lausanne. Handedness was assessed through informal verbal inquiry. All participants gave informed consent prior to participation and were fully debriefed after the experiment, according to the guidelines of the local ethics committee. The study was conducted in accordance with the declaration of Helsinki.

\section{Stimuli}

As stimuli we used pictures representing a human avatar in different orientations (see Fig. 1; cf. Tadi et al., 2009). The avatar was rotated along the yaw axis in steps of $45^{\circ}$, where $0^{\circ}$ was defined as the avatar being viewed on the back and $180^{\circ}$ as facing the avatar. The avatar could be rotated in a clockwise direction with respect to the $0^{\circ}$ position $\left(+45^{\circ}\right.$, $+90^{\circ},+135^{\circ}$ ) or counter-clockwise with respect to the $0^{\circ}$ position $\left(-45^{\circ},-90^{\circ},-135^{\circ}\right)$. In $50 \%$ of the stimuli the left hand of the avatar had a different color than the avatar's body, and in the other half of the stimuli the right hand was colored differently. Thus, in total 16 different stimuli were used.

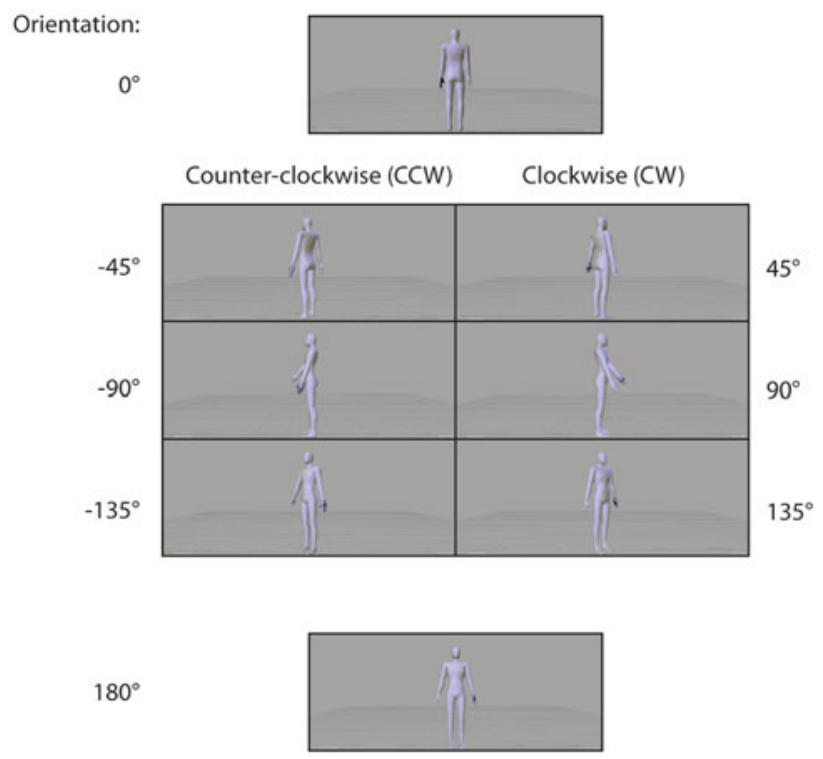

Fig. 1 Example stimuli used in the experiment, where $0^{\circ}$ was defined as the avatar being viewed on the back and $180^{\circ}$ as facing the avatar. The avatar could be rotated in a counter-clockwise direction with respect to the $0^{\circ}$ position $\left(-45^{\circ},-90^{\circ},-135^{\circ}\right.$; see left middle panel) or a clockwise direction with respect to the $0^{\circ}$ position $\left(+45^{\circ}\right.$, $+90^{\circ},+135^{\circ}$; see right middle panel) 


\section{Experimental setup}

The experiment was conducted in complete darkness in a sound-shielded room in which a human motion platform was placed (see Fig. 2a) (see also van Elk \& Blanke, 2012). A chair was mounted on a beam platform (diameter $=200 \mathrm{~cm}$ ) fixed on an electrical engine. The electrical engine was digitally servo-controlled (PCI-7352) and its software controller allowed for a precise positioning $\left( \pm 0.01^{\circ}\right)$ and for the execution of rotation profiles expressed as sequences of positions at $100 \mathrm{~Hz}$. All rotations were carried out around the yaw axis. A $22^{\prime \prime}$ computer screen was mounted on the chair with an adjustable but fixed arm, placed at $40 \mathrm{~cm}$ away from the subject's eyes. Images were generated by an on-board computer, which was controlled from the outside by network desktop sharing. A rumble pad PC game controller (Saitek P2600) was connected to the computer to measure participants' responses.

Participants were seated in the chair wearing seatbelts, with their head aligned to their body's $z$ axis and precisely located in the center of rotation. An infrared surveillance camera was mounted on the chair showing the face of the participants and allowed to monitor participants' eye movements online. Another infrared camera displayed the chair itself. During the experiment communication was possible between the subject and the experimenter.

\section{Experimental procedure}

In the experiment participants performed a MBT task and indicated whether the left or the right hand of an avatar had a different color than the avatar's body, by pressing, respectively, the left or the right button of the game controller. They were told that the avatar would be displayed in different positions and that they should judge the laterality as seen from the avatar's perspective. No explicit instruction was given as to how participants should solve the task (e.g., by rotating their own body in the position of the avatar, or by rotating the picture on the screen to the position of their own body). At the beginning of the experiment participants practiced while the chair was standing still. The experiment started after it was established that the participants understood the task.

An overview of the events during each trial is represented in Fig. 2b. The chair accelerated during 2,000 ms to a speed of $45 \%$ and $500 \mathrm{~ms}$ after acceleration onset the stimulus (i.e., picture of the avatar) was presented, which remained on the screen for $1,500 \mathrm{~ms}$ or until the subject responded. The chair rotated at a constant speed during 2,000-3,000 ms, followed by a $2,000 \mathrm{~ms}$ deceleration to $0 \% \mathrm{~s}$. $500 \mathrm{~ms}$ after the onset of the deceleration a different stimulus was presented on the screen for 1,500 ms or until the subject responded. Thus, stimuli were presented both during the acceleration and the deceleration phase of the chair.

During each block the motion platform generated 16 clockwise and 16 counter-clockwise rotations in a random order. Each rotation profile consisted of four phases starting with $2,000 \mathrm{~ms}$ acceleration to a speed of $45 \%$, 2,000-3,000 ms of constant speed, 2,000 ms deceleration to $0 \%$, followed by a $2,000-3,000 \mathrm{~ms}$ no-movement interval. The rotation profile and speed were based on own pilot studies with the present paradigm showing that this speed provided an optimum between generating robust vestibular sensations, without inducing motion sickness. The constant movement and no-movement interval varied pseudo-randomly between 2,000 and 3,000 ms in steps of
Fig. 2 Experimental setup and procedure. a Overview of the experimental setup. The participant was seated on a motion platform allowing passive full body rotations. The participant was facing a screen that was attached to the chair and on which the stimuli for the experimental task were displayed. b Overview of the experimental procedure. Each trial started with a fixation cross being presented for 2,000-3,000 ms, followed by the onset and offset of the chair rotation. Pictures were presented during the acceleration and deceleration phase and participants responded to the pictures by making a left/right button press
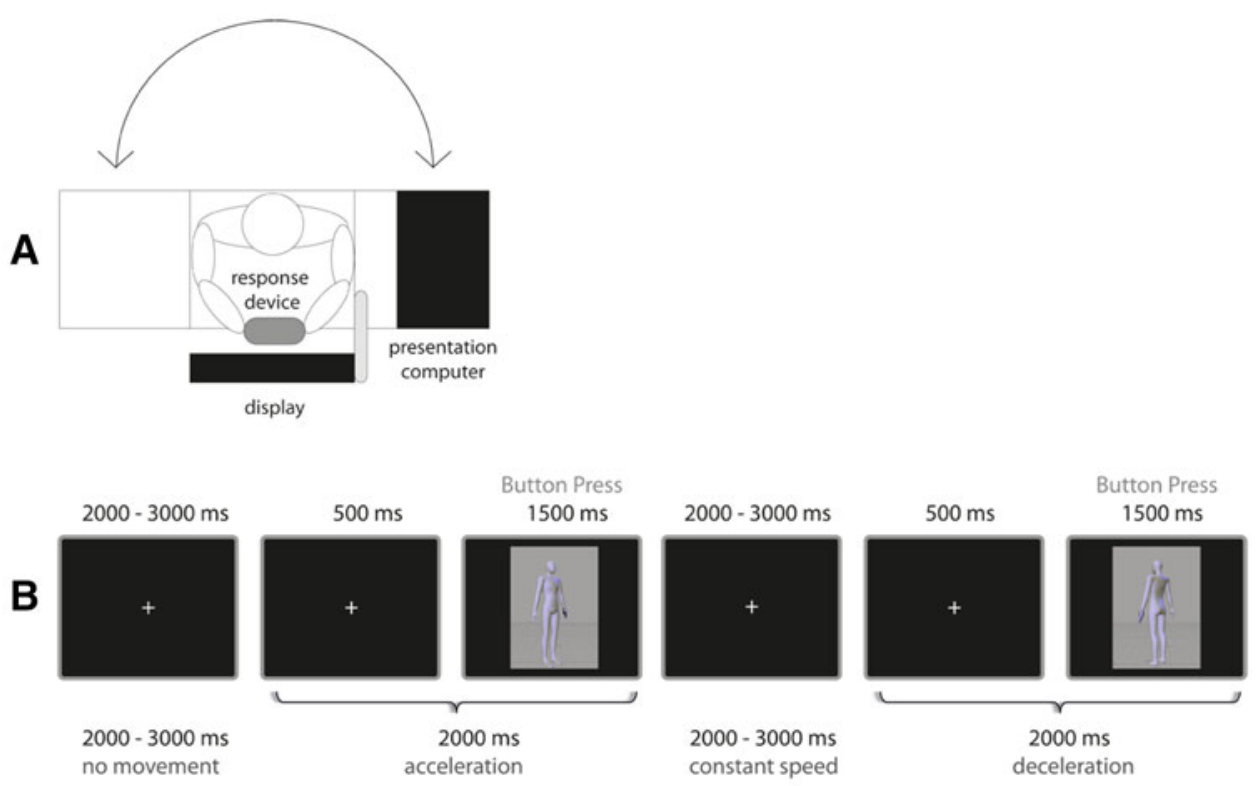
$100 \mathrm{~ms}$ to prevent the subject from anticipating the onset and offset of the rotations. For each subject and for each block a different 5 min rotation profile was generated. In ten different blocks participants were required to respond to the pictures on the screen while they received passive whole-body rotations along the yaw axis.

During the experiment white noise was presented over the participants' headphones and a black blanket covered the chair, to avoid the participant from inferring the rotation direction based on auditory or visual cues (residual light emanating from the stimulus display). Stimulus presentation and button press responses were controlled and stored for offline analysis using Presentation software version 12.2 (Neurobehavioral Systems, Davis, CA).

At the end of the experiment participants completed a short questionnaire, to assess the effects of vestibular rotation (simulator sickness questionnaire (SSQ); cf. Kennedy, Lane, Berbaum, \& Lilienthal, 1989), the strategy participants used for the mental imagery task (i.e., imagining one's own body rotating vs. imagining the avatar's body rotating; cf. Lenggenhager et al., 2008) and a questionnaire to assess their overall spatial and object imagery abilities (object spatial imagery questionnaire (OSIQ); cf. Blajenkova, Kozhevnikov, \& Motes, 2006). The OSIQ measures participant's tendency and experience to use object and spatial visual imagery, ranging from 1 (=very low imagery ability) to 5 (=very high imagery ability). The object imagery subscale assesses one's preferences for representing and processing colorful, pictorial and detailed images of individual objects. The spatial imagery subscale assesses one's preferences for representing and processing schematic images, spatial relations between objects and spatial transformations. In total the experiment took about $1.5 \mathrm{~h}$.

\section{Data analysis}

For the main analysis, reaction times exceeding the participant's mean by more than 2 standard deviations were excluded from analysis. For each participant the mean reaction time was calculated per stimulus category according to the rotation direction of the chair (CW vs. $\mathrm{CCW})$. For the analysis we were interested in the stimuli in which the position of the avatar implied a specific rotation direction and that could accordingly be classified as implying a $\mathrm{CW}$ or $\mathrm{CCW}$ rotation. Thus, reaction times were analyzed using a 2 (chair direction: $\mathrm{CW}$ vs. $\mathrm{CCW}) \times 2$ (stimulus direction: $\mathrm{CW}$ vs. $\mathrm{CCW}) \times 3$ (stimulus orientation: $\left.45^{\circ}, 90^{\circ}, 135^{\circ}\right)$ repeated measures ANOVA. This analysis was conducted separately for stimuli presented during the acceleration phase or the deceleration phase.

In addition, for each participant the mean ratings on the SSIQ and on the object and spatial subscales of the OSIQ were calculated (after reverse coding of the negatively formulated items).

\section{Results}

Questionnaire data

At the end of the experiment ten participants indicated that they imagined rotating the avatar on the screen and eight participants indicated that they imagined rotating their own body. Two participants reported an explicit strategy for the $0^{\circ}$ and the $180^{\circ}$ stimuli (i.e., responding congruent if the avatar was facing away, responding incongruent if the avatar was facing toward the participant).

The average score on the object imagery abilities subscale was $3.3(\mathrm{SD}=0.57)$ and on the spatial imagery abilities subscale $3.1 \quad(\mathrm{SD}=0.46)$. The SSQ indicated that on average participants experienced none to very mild symptoms during the experiment (average $=1.7$, $\mathrm{SD}=0.38$; with $1=$ none, $2=$ slight, $3=$ moderate, $4=$ severe). The most reported symptoms included fatigue (average $=2.5$ ), eyestrain (average $=2.2$ ) and difficulty focusing (average $=2.1$ ).

\section{Reaction times}

Errors were made in only $1.5 \%$ of all trials and were not further analyzed. Reaction times are represented in Fig. 3. The analysis of reaction times for all participants during the acceleration phase showed a main effect of stimulus orientation, $F(2,34)=12.5, p<0.001, \eta^{2}=0.42$, reflecting increased reaction times for stimuli with increased angular disparity (i.e., the avatar's position differs more strongly from the participant's position). An interaction was found between chair direction and stimulus direction, $F(1$, $17)=7.7, p<0.05, \eta^{2}=0.31$, reflecting that for $\mathrm{CW}$ chair rotations participants tended to respond faster to stimuli implying a $\mathrm{CW}$ rotation $(641 \mathrm{~ms})$ compared to a $\mathrm{CCW}$ rotation $(651 \mathrm{~ms})$, whereas for $\mathrm{CCW}$ chair rotations participants tended to respond faster to stimuli implying a $\mathrm{CCW}$ rotation $(643 \mathrm{~ms})$ compared to a $\mathrm{CW}$ rotation $(654 \mathrm{~ms}) .^{2}$ No other effects were found significant. For the analysis of reaction times during the deceleration phase

\footnotetext{
${ }^{2}$ Post hoc tests did not reveal significant differences between stimuli implying a CW vs. a CCW rotation $(t(17)=1.4, p=0.18$ for $\mathrm{CW}$ chair rotations, $t(17)=-1.6, p=0.14$ for $\mathrm{CCW}$ chair rotations), indicating that although rotation direction did affect mental transformation, it did not result in a complete reversal of the directionality effects. This could be partly related to the fact of spatial compatibility effects partly underlying the MBT task (Gardner \& Potts, 2011; May \& Wendt, 2012) and strategy differences between participants (i.e., egocentric vs. allocentric strategy; see also below).
} 

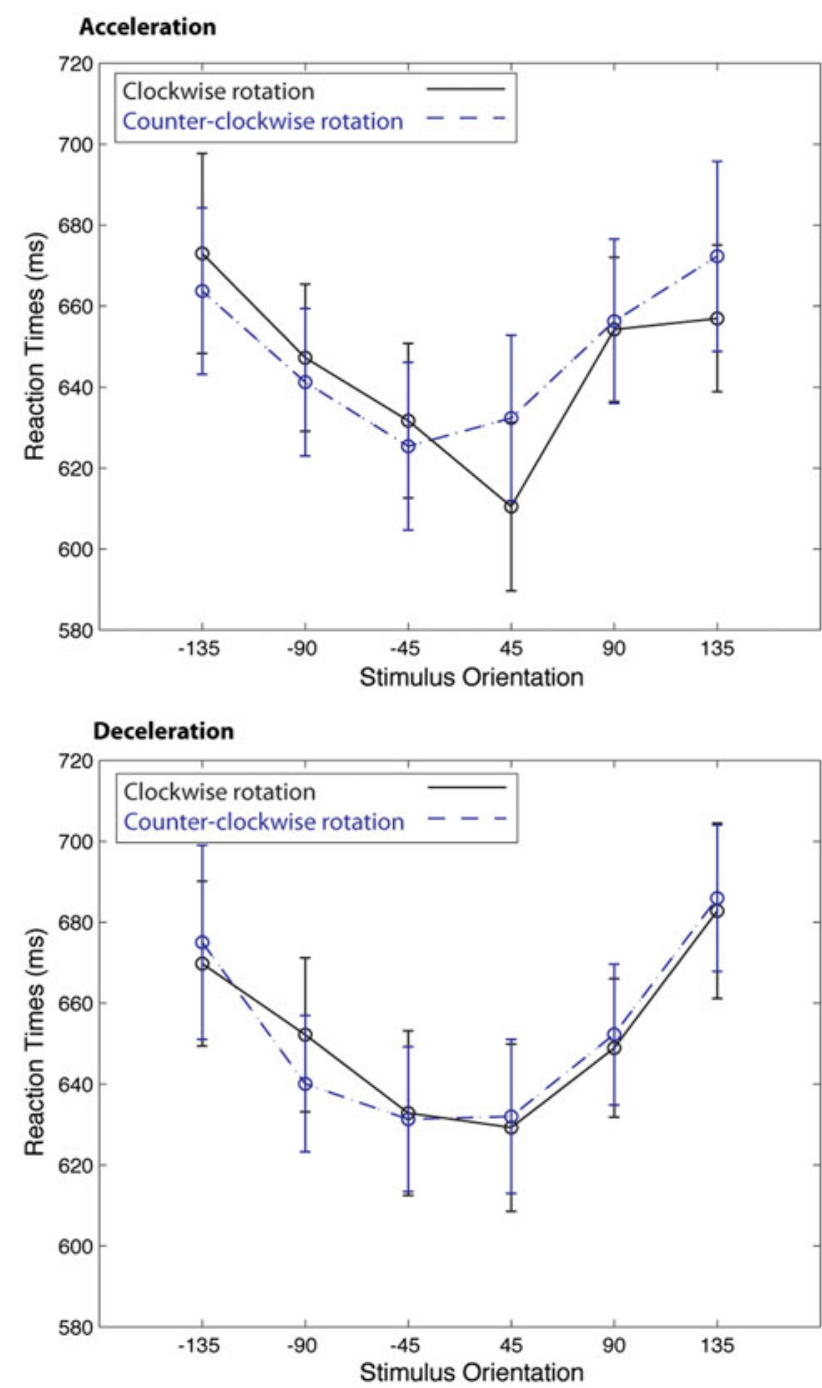

Fig. 3 Behavioral results. Reaction times according to stimulus orientation during acceleration (upper graph) and deceleration (lower graph). Black solid lines represent clockwise chair rotations and blue dotted lines represent counter-clockwise rotations. Error bars represent standard errors

only a main effect of stimulus orientation was observed, reflecting slower reaction times with increased angular disparity, $F(2,34)=17.9, p<0.001, \eta^{2}=0.51$, but no other effects were found significant (Fig. 3).

\section{Control for spatial compatibility effects}

The stimuli that were used in the experiment were characterized by strong spatial features (i.e., colored hand of the avatar appearing on the left or the right side of the screen). In addition, participants responded by making a left-right button press and the chair rotated in a clockwise or a counter-clockwise direction. Accordingly, a possible confound may be that the facilitatory effect of chair rotation on MBT was modulated by spatial compatibility
Table 1 Reaction times according to the rotation direction of the chair (CW: left column, CCW: right column) and the movement phase of the chair (upper panel: acceleration, lower panel: deceleration). Reaction times were averaged according to whether the color cue appeared at the left or the right side of the screen and according to whether participants responded by making a left or a right button press. Stimulus-response compatibility effects are reflected in faster responses when the color cue appears at the same location as the response hand (e.g., 'color cue left'-'response hand left')

\begin{tabular}{llllll}
\hline Chair direction & CW & & & CCW \\
Response hand & Left & Right & & Left & Right \\
\hline Acceleration & & & & & \\
$\quad \begin{array}{lllll}\text { Color cue left } \\
\text { Color cue right }\end{array}$ & $629(21)$ & $697(21)$ & & $638(21)$ & $691(20)$ \\
Deceleration & & $610(29)$ & $685(20)$ & $618(20)$ \\
Color cue left & $641(22)$ & $698(18)$ & $636(18)$ & $705(19)$ \\
Color cue right & $694(20)$ & $622(21)$ & $684(20)$ & $624(18)$ \\
\hline
\end{tabular}

effects between the stimulus, the response hand and the chair.

We checked for spatial compatibility effects between the stimulus, the response hand and the chair direction. To this end, each stimulus was categorized according to whether the colored hand of the avatar appeared on the left or the right side of the screen (except for the $90^{\circ}$ stimuli that could not be clearly categorized according to the spatial position of the colored hand; see Fig. 1). Subsequently, reaction times were analyzed using a 2 (color cue: left vs. right side) $\times 2$ (response hand: left vs. right) $\times 2$ (chair direction: $\mathrm{CW}$ vs. $\mathrm{CCW}$ ) repeated measures ANOVA. The behavioral data for this analysis are represented in Table 1.

For reaction times during the acceleration phase, a main effect of color cue, $F(1,17)=7.8, p<0.05, \eta^{2}=37$, indicated faster responses to stimuli in which the colored hand appeared at the left side $(637 \mathrm{~ms})$ compared to the right side $(647 \mathrm{~ms})$. A main effect of chair direction, $F(1,17)=4.5, p<0.05, \eta^{2}=21$, reflected that reaction times were slightly faster for $\mathrm{CW}(653 \mathrm{~ms})$ compared to $\mathrm{CCW}$ rotations $(658 \mathrm{~ms})$. A significant interaction between color cue and response hand, $F(1,17)=42.6, p<0.001$, $\eta^{2}=72$, reflected a spatial stimulus-response compatibility effect. When the colored hand of the avatar appeared at the left side of the screen, participants responded faster by pressing the left button $(633 \mathrm{~ms})$ compared to the right button $(694 \mathrm{~ms})$, but when the colored hand appeared at the right side of the screen participants responded faster by pressing the right button (614 ms) compared to the left button $(681 \mathrm{~ms})$. Importantly, no significant interactions were observed between chair direction, color cue and response hand $(p s>0.27)$, indicating that the rotation 
direction of the chair did not modulate the spatial compatibility effects.

Analysis of reaction times during the deceleration phase indicated an interaction between color cue and response hand, $F(1,17)=53.2, p<0.001, \eta^{2}=0.76$, reflecting stimulus-response compatibility effects similar to the acceleration phase. That is, when the colored hand appeared at the left side of the screen participants responded faster with a left button press (639 ms) compared to a right button press $(701 \mathrm{~ms})$, but when the colored hand appeared at the right side of the screen participants responded faster with a right button press $(623 \mathrm{~ms})$ compared to a left button press $(689 \mathrm{~ms})$. The interaction between chair direction and response hand was non-significant, $F(1,17)=3.1, p=0.1$, but reflected a tendency for faster responses when the response button was congruent with the rotation direction of the chair. Importantly, no significant interactions were observed between chair direction, color cue and response hand ( $p$ 's $>0.56$ ), indicating that the rotation direction of the chair did not modulate the spatial compatibility effects.

\section{Post hoc analyses}

In a post hoc analysis, we were interested in the question whether the observed effect between chair rotation and implied rotation was modulated by the strategy that participants used for the mental imagery task (i.e., imagining one's own body rotating or imagining the avatar rotating). To this end we used the strategy that participants reported for the mental imagery task (i.e., own body vs. other body rotation) as a between-participants variable in the repeated measures ANOVA. For reaction times during the acceleration phase, a trend toward a significant interaction effect was observed between strategy, chair direction and stimulus direction, $F(1,16)=3.7, p=0.07, \eta^{2}=0.19$. Post hoc ANOVAs for the two groups of participants revealed that participants who imagined their own body rotating showed an interaction between chair direction and stimulus direction, $F(1,7)=13.1, p<0.01$ (see upper graph of Fig. 4), while for participants who imagined the avatar rotating no interaction was found $(F<1)$. For reaction times during the deceleration phase no significant interactions with Strategy were observed $(F(2,32)=1.9$, n.s. $)$.

\section{Discussion}

In the present study we hypothesized that vestibular stimulation, due to passive own-body displacements, would modulate spatial perspective taking as measured by a MBT task. A congruency effect was observed, reflected in faster reaction times if the direction of the implied mental body
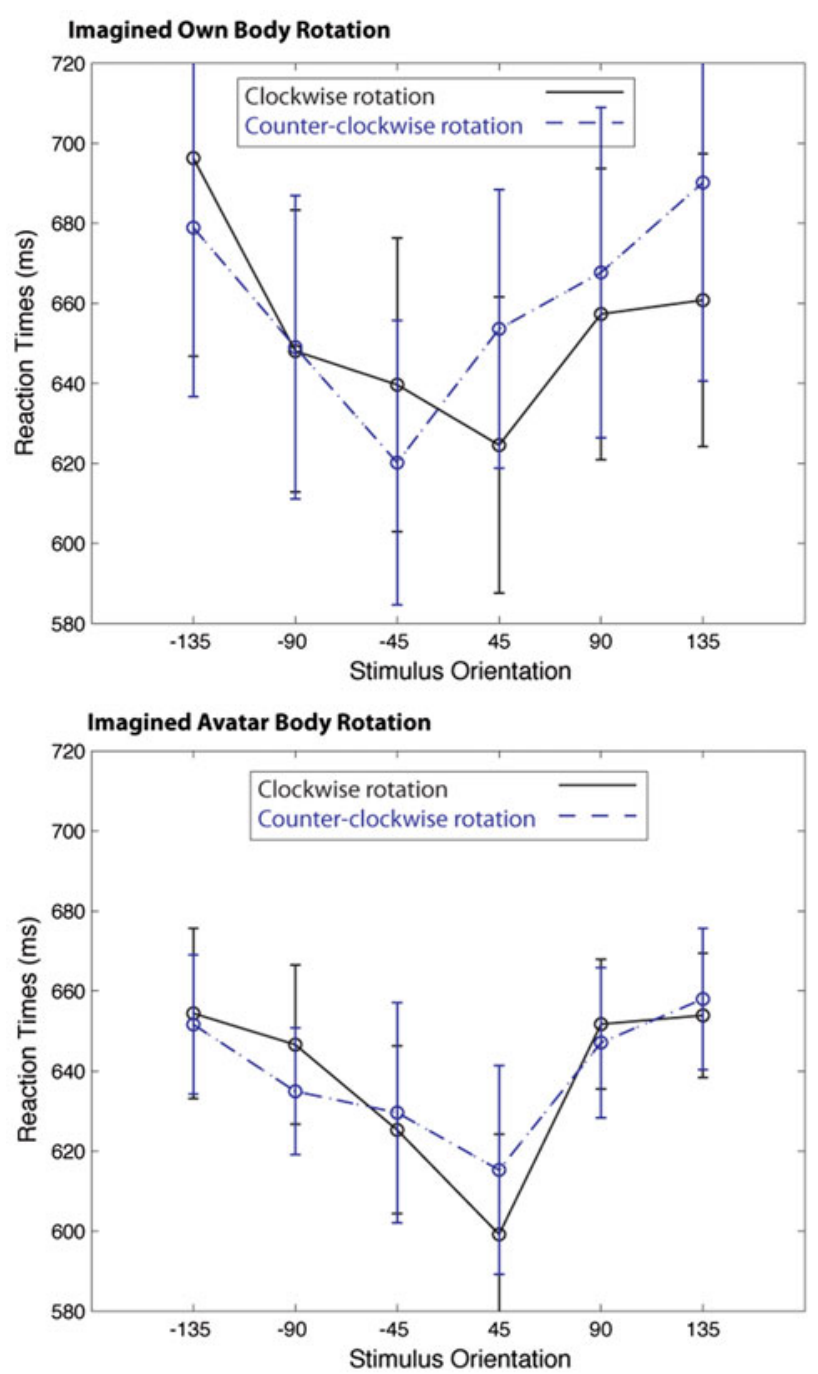

Fig. 4 Reaction times according to stimulus orientation during acceleration for participants who imagined their own body rotating (upper graph) or who imagined the avatar's body rotating (lower graphs). Black solid lines represent clockwise chair rotations and blue dotted lines represent counter-clockwise rotations. Error bars represent standard errors

rotation matched the direction of the actual body rotation on the human motion platform.

The mental-vestibular congruency effect between the imagined body rotation direction and the actual body rotation direction indicates a selective effect of vestibular stimulation on imagined body transformations. Only when the imagined and the actual body rotation were in the same direction, imagined body transformation was facilitated, as reflected in faster reaction times. A previous study has used artificial vestibular stimulations and showed a general and non-direction-specific effect of galvanic stimulation on imagined rotation (Lenggenhager et al., 2008). In another study it was found that caloric stimulation that mimics vestibular signals of a rightward head rotation specifically 
facilitated rightward clockwise mental rotations (Falconer \& Mast, 2012). The present study extends these data by physiologically stimulating the vestibular organs through full body rotations and indicates that direction-specific vestibular information, as detected by the semicircular canals and analyzed by the central vestibular system, can facilitate MBTs.

Over the last decades, many studies have shown that spatial perspective taking involves the integration of both motor (Huttenlocher \& Presson, 1979; Kozhevnikov \& Hegarty, 2001; Zacks \& Michelon, 2005) and proprioceptive information (Huttenlocher \& Presson, 1979; Kessler \& Rutherford, 2010; Kessler \& Thomson, 2010; Kozhevnikov \& Hegarty, 2001; Tversky \& Hard, 2009; Zacks \& Michelon, 2005). For instance, in a recent study a facilitatory effect of one's body posture on spatial perspective taking has been reported (Kessler \& Thomson, 2010). These findings provide support for the notion that spatial perspective taking is an embodied transformation process, whereby a self-initiated emulation of a bodily movement or displacement is used to adopt the imposed spatial perspective. The direction-specific effect observed in the present experiment extends this notion by showing that vestibular signals may facilitate the spatial updating of one's body representation, likely via a process of multisensory integration. More specifically, the facilitatory effect of vestibular stimulation may reflect the integration of low-level vestibular signals regarding self-rotation with a high-level multimodal representation of one's body (Blanke, 2012), possibly mediated by multisensory brain areas like the parieto-insular vestibular cortex (Lopez \& Blanke, 2011), the posterior part of the superior temporal gyrus (Ionta, Gassert, \& Blanke, 2011; Ionta et al., 2011), as well as regions with vestibular neurons in posterior parietal and premotor cortex (Lopez \& Blanke, 2011; Petkova et al., 2011).

Participants who reported a strategy whereby they rotated their own body ('egocentric mental rotation strategy') tended to show a stronger direction-specific vestibular influence on imagined own-body rotation, than participants who reported that they imagined rotating the avatar on the screen ('allocentric mental rotation strategy'). This finding is in line with a study by Kessler and Thomson (2010) showing an effect of body posture on mental rotation only during self-rotation but not during object rotation, suggesting that egocentric and allocentric rotation may be based on different embodied simulation processes. Similarly, the facilitatory effect of the congruency between real and imagined rotations that is further modulated by the imagery modality-present for egocentric-based kinesthetic imagery but not for allocentric-based visual imagery-demonstrates the interdependence between proprioception and mental imagery (Fourkas, Ionta, \& Aglioti, 2006). Our results extend this previous body of evidence suggesting that individual differences in the strategy used for spatial perspective taking have a modulating effect on the mentalvestibular effects in addition to mental-proprioceptive interactions.

We note that the absence of an effect of vestibular stimulation during the deceleration phase was unexpected, given that the signals produced by the vestibular organs do not distinguish between deceleration in a specific direction and acceleration in the opposite direction. Different reasons may account for this. First, the fact that no effect was found during the deceleration phase may be related to aftereffects due to the preceding acceleration, interfering with or even cancelling out the effects of the deceleration (Lackner \& Graybiel, 1977). The vestibular after-effects were a natural consequence of the fact that the inter-trial interval was relatively short (i.e., 2 to $3 \mathrm{~s}$ ) in order to reduce the total duration of the experiment. Furthermore, several studies have shown that mental rotation and imagined body transformations are strongly modulated by top-down factors, such as task instruction or attention (Arzy, Thut, Mohr, Michel, \& Blanke, 2006; Blanke et al., 2005; Sirigu \& Duhamel, 2001; Viswanathan, Fritz, \& Grafton, 2012). Accordingly, in the present study cognitive factors related to the conscious representation of movement may have interfered with the integration of vestibular signals. That is, knowing that one is rotating and then decelerating is different from knowing that one is not rotating and then accelerating. This high-level knowledge may have modulated the effects of vestibular stimulation on imagined body rotations.

Previous studies have suggested that spatial attention is strongly coupled to vestibular mechanisms, such that passive own-body displacements result in an automatic shift of attention in the rotation direction of one's body (Figliozzi, Guariglia, Silvetti, Siegler, \& Doricchi, 2005; Karnath \& Dieterich, 2006). The MBT task used in the present study had a strong spatial component (i.e., colored hand of the avatar appearing at the left or the right side of the screen). Recent studies suggest that both mental transformations and spatial (in)compatibility effects may underlie the MBT task (Gardner \& Potts, 2011; May \& Wendt, 2012) and also in the present study a strong spatial compatibility effect was observed reflected in faster responses if the colored hand was at the same spatial side as the hand used for responding. However, no relation was observed between the location of the stimulus cue, the response hand and the rotation direction of the chair, thereby ruling out the possible confound that spatial compatibility effects could underlie the selective vestibular influence on spatial perspective taking. Thereby this study supports the idea that different processes may be involved in spatial perspective taking and that in addition to spatial compatibility effects, the mental transformation effort reflects an embodied 
process that is unrelated to the response modality that is used (see also: Kessler \& Rutherford, 2010).

In summary, this study supports the idea that spatial perspective taking is an embodied process that involves transformations of the participant's own body. Whereas previous studies have highlighted the role of integrating motor signals and proprioceptive information (Amorim et al., 2006; Creem-Regehr, Neil, \& Yeh, 2007; Keehner, Guerin, Miller, Turk, \& Hegarty, 2006; Kessler \& Thomson, 2010; Parsons, 1987; Vogeley et al., 2004; Wraga, 2003; Zacks \& Michelon, 2005), this study underlines the central importance of vestibular information for embodied perspective taking. These signals may be of particular importance for mental imagery with respect to one's entire body as opposed to imagery for body parts (Parsons, 1987) where motor and proprioceptive mechanisms may predominate. Finally, these data contribute to the growing number of studies showing that vestibular signals do not only support balance, locomotion, and space perception, but are also involved in cognitive aspects of own-body representations (Lopez et al., 2008), bodily self consciousness (Blanke, 2012), and bistable visual perception (van Elk \& Blanke, 2012).

Acknowledgments The present study was supported by a Marie Curie Grant (IEF grant 252713), the Virtual Embodiment and Robotic Re-Embodiment grant within the European FP7 framework (VERE grant 257695) and the Swiss Science Foundation (SINERGIA CRSII1-125135/1).

\section{References}

Amorim, M. A., Isableu, B., \& Jarraya, M. (2006). Embodied spatial transformations: "body analogy" for the mental rotation of objects. Journal of Experimental Psychology: General, 135(3), 327-347.

Angelaki, D. E., \& Cullen, K. E. (2008). Vestibular system: the many facets of a multimodal sense. Annual Review of Neuroscience, $31,125-150$.

Arzy, S., Thut, G., Mohr, C., Michel, C. M., \& Blanke, O. (2006). Neural basis of embodiment: distinct contributions of temporoparietal junction and extrastriate body area. Journal of Neuroscience, 26(31), 8074-8081.

Avraamides, M. N., \& Kelly, J. W. (2008). Multiple systems of spatial memory and action. Cognitive Processes, 9(2), 93-106.

Blajenkova, O., Kozhevnikov, M., \& Motes, A. M. (2006). Objectspatial imagery: a new self-report imagery questionnaire. Applied Cognitive Psychology, 20(2), 239-263.

Blanke, O. (2012). Multisensory brain mechanisms of bodily selfconsciousness. Nature Reviews Neuroscience, 13(8), 556-571.

Blanke, O., Ionta, S., Fornari, E., Mohr, C., \& Maeder, P. (2010). Mental imagery for full and upper human bodies: common right hemisphere activations and distinct extrastriate activations. Brain Topography, 23(3), 321-332.

Blanke, O., Mohr, C., Michel, C. M., Pascual-Leone, A., Brugger, P., Seeck, M., et al. (2005). Linking out-of-body experience and self processing to mental own-body imagery at the temporoparietal junction. Journal of Neuroscience, 25(3), 550-557.
Creem-Regehr, S. H. (2003). Updating space during imagined selfand array translations. Memory and Cognition, 31(6), 941-952.

Creem-Regehr, S. H., Neil, J. A., \& Yeh, H. J. (2007). Neural correlates of two imagined egocentric transformations. Neuroimage, 35(2), 916-927.

Easton, R. D., \& Sholl, M. J. (1995). Object-array structure, frames of reference, and retrieval of spatial knowledge. Journal of Experimental Psychology. Learning, Memory, and Cognition, 21(2), 483-500.

Falconer, C. J., \& Mast, F. W. (2012). Balancing the mind. Experimental Psychology, 59(6), 332-339.

Ferre, E. R., Bottini, G., \& Haggard, P. (2011). Vestibular modulation of somatosensory perception. European Journal of Neuroscience, 34(8), 1337-1344.

Figliozzi, F., Guariglia, P., Silvetti, M., Siegler, I., \& Doricchi, F. (2005). Effects of vestibular rotatory accelerations on covert attentional orienting in vision and touch. Journal of Cognitive Neuroscience, 17(10), 1638-1651.

Fourkas, A. D., Ionta, S., \& Aglioti, S. M. (2006). Influence of imagined posture and imagery modality on corticospinal excitability. Behavioral Brain Research, 168(2), 190-196.

Gardner, M. R., \& Potts, R. (2011). Domain general mechanisms account for imagined transformations of whole body perspective. Acta Psychologica, 137(3), 371-381.

Huttenlocher, J., \& Presson, C. C. (1979). The coding and transformation of spatial information. Cognitive Psychology, 11(3), 375-394.

Ionta, S., Gassert, R., \& Blanke, O. (2011a). Multi-sensory and sensorimotor foundation of bodily self-consciousness-an interdisciplinary approach. Frontiers in Psychology, 2, 383.

Ionta, S., Heydrich, L., Lenggenhager, B., Mouthon, M., Fornari, E., Chapuis, D., et al. (2011b). Multisensory mechanisms in temporo-parietal cortex support self-location and first-person perspective. Neuron, 70(2), 363-374.

Karnath, H. O., \& Dieterich, M. (2006). Spatial neglect—a vestibular disorder? Brain, 129(Pt 2), 293-305.

Keehner, M., Guerin, S. A., Miller, M. B., Turk, D. J., \& Hegarty, M. (2006). Modulation of neural activity by angle of rotation during imagined spatial transformations. Neuroimage, 33(1), 391-398.

Kennedy, R. S., Lane, N. E., Berbaum, K. S., \& Lilienthal, M. G. (1989). Simulator sickness questionnaire: an enhanced method for quantifying simulator sickness. The International Journal of Aviation Psychology, 3(3), 203-220.

Kessler, K., \& Rutherford, H. (2010). The two forms of visuo-spatial perspective taking are differently embodied and subserve different spatial prepositions. Frontiers in Psychology, 1, 213.

Kessler, K., \& Thomson, L. A. (2010). The embodied nature of spatial perspective taking: embodied transformation versus sensorimotor interference. Cognition, 114(1), 72-88.

Kessler, K., \& Wang, H. (2012). Spatial perspective taking is an embodied process, but not for everyone in the same way: differences predicted by sex and social skills score. Spatial Cognition and Computation: An Interdisciplinary Journal, 12, 133-158.

Kozhevnikov, M., \& Hegarty, M. (2001). A dissociation between object manipulation spatial ability and spatial orientation ability. Memory and Cognition, 29(5), 745-756.

Lackner, J. R., \& Graybiel, A. (1977). Somatosensory motion aftereffect following earth-horizontal rotation about the Z-axis: a new illusion. Aviation, Space and Environmental Medicine, 48(6), 501-502.

Lenggenhager, B., Lopez, C., \& Blanke, O. (2008). Influence of galvanic vestibular stimulation on egocentric and object-based mental transformations. Experimental Brain Research, 184(2), 211-221. 
Lopez, C., Bachofner, C., Mercier, M., \& Blanke, O. (2009). Gravity and observer's body orientation influence the visual perception of human body postures. Journal of Vision, 9(5), 1.1-14.

Lopez, C., \& Blanke, O. (2011). The thalamocortical vestibular system in animals and humans. Brain Research Reviews, 67(1-2), 119-146.

Lopez, C., Halje, P., \& Blanke, O. (2008). Body ownership and embodiment: vestibular and multisensory mechanisms. Clinical Neurophysiology, 38(3), 149-161.

Lopez, C., Heydrich, L., Seeck, M., \& Blanke, O. (2010a). Abnormal self-location and vestibular vertigo in a patient with right frontal lobe epilepsy. Epilepsy and Behavior, 17(2), 289-292.

Lopez, C., Lenggenhager, B., \& Blanke, O. (2010b). How vestibular stimulation interacts with illusory hand ownership. Consciousness and Cognition, 19(1), 33-47.

May, M. (2004). Imaginal perspective switches in remembered environments: transformation versus interference accounts. Cognitive Psychology, 48(2), 163-206.

May, M., \& Wendt, M. (2012). Separating mental transformations and spatial compatibility effects in the own body transformation task. Cognitive Processing, 13(Suppl 1), S257-S260.

Mohr, C., Blanke, O., \& Brugger, P. (2006). Perceptual aberrations impair mental own-body transformations. Behavioral Neuroscience, 120(3), 528-534.

Moll, H., \& Meltzoff, A. N. (2011). How does it look? Level 2 perspective-taking at 36 months of age. Child Development, 82(2), 661-673.

Parsons, L. M. (1987). Imagined spatial transformation of one's body. Journal of Experimental Psychology: General, 116(2), 172-191.

Petkova, V. I., Bjornsdotter, M., Gentile, G., Jonsson, T., Li, T. Q., \& Ehrsson, H. H. (2011). From part- to whole-body ownership in the multisensory brain. Current Biology, 21(13), 1118-1122.

Rieser, J. J. (1989). Access to knowledge of spatial structure at novel points of observation. Journal of Experimental Psychology. Learning, Memory, and Cognition, 15(6), 1157-1165.

Rieser, J. J., Garing, A. E., \& Young, M. F. (1994). Imagery, action, and young children's spatial orientation: it's not being there that counts, it's what one has in mind. Child Development, 65(5), $1262-1278$.
Rieser, J. J., Guth, D. A., \& Hill, E. W. (1986). Sensitivity to perspective structure while walking without vision. Perception, 15(2), 173-188.

Shinder, M. E., \& Taube, J. S. (2010). Differentiating ascending vestibular pathways to the cortex involved in spatial cognition. Journal of Vestibular Research, 20(1), 3-23.

Sirigu, A., \& Duhamel, J. R. (2001). Motor and visual imagery as two complementary but neurally dissociable mental processes. Journal of Cognitive Neuroscience, 13(7), 910-919.

Sodian, B., Thoermer, C., \& Metz, U. (2007). Now I see it but you don't: 14-month-olds can represent another person's visual perspective. Developmental Science, 10(2), 199-204.

Tadi, T., Overney, L. S., \& Blanke, O. (2009). Three sequential brain activations encode mental transformations of upright and inverted human bodies: a high resolution evoked potential study. Neuroscience, 159(4), 1316-1325.

Tversky, B., \& Hard, B. M. (2009). Embodied and disembodied cognition: spatial perspective-taking. Cognition, 110(1), 124-129.

van Elk, M., \& Blanke, O. (2012). Balancing bistable perception during self-motion. Experimental Brain Research, 222(3), 219-228.

Viswanathan, S., Fritz, C., \& Grafton, S. T. (2012). Telling the right hand from the left hand: multisensory integration, not motor imagery, solves the problem. Psychological Science, 23(6), 598-607.

Vogeley, K., May, M., Ritzl, A., Falkai, P., Zilles, K., \& Fink, G. R. (2004). Neural correlates of first-person perspective as one constituent of human self-consciousness. Journal of Cognitive Neuroscience, 16(5), 817-827.

Wraga, M. (2003). Thinking outside the body: an advantage for spatial updating during imagined versus physical self-rotation. Journal of Experimental Psychology. Learning, Memory, and Cognition, 29(5), 993-1005.

Wraga, M., Shephard, J. M., Church, J. A., Inati, S., \& Kosslyn, S. M. (2005). Imagined rotations of self versus objects: an fMRI study. Neuropsychologia, 43(9), 1351-1361.

Zacks, J. M., \& Michelon, P. (2005). Transformations of visuospatial images. Behavioral and Cognitive Neuroscience Reviews, 4(2), 96-118. 